\title{
Concatenated coding system with iterated sequential inner decoding
}

\author{
Jensen, Ole Riis; Paaske, Erik
}

Published in:

Proceedings of the IEEE International Symposium on Information Theory

Link to article, DOI:

10.1109/ISIT.1995.550456

Publication date:

1995

Document Version

Publisher's PDF, also known as Version of record

Link back to DTU Orbit

Citation (APA):

Jensen, O. R., \& Paaske, E. (1995). Concatenated coding system with iterated sequential inner decoding. In Proceedings of the IEEE International Symposium on Information Theory (pp. 469-469). IEEE.

https://doi.org/10.1109/ISIT.1995.550456

\section{General rights}

Copyright and moral rights for the publications made accessible in the public portal are retained by the authors and/or other copyright owners and it is a condition of accessing publications that users recognise and abide by the legal requirements associated with these rights.

- Users may download and print one copy of any publication from the public portal for the purpose of private study or research.

- You may not further distribute the material or use it for any profit-making activity or commercial gain

- You may freely distribute the URL identifying the publication in the public portal

If you believe that this document breaches copyright please contact us providing details, and we will remove access to the work immediately and investigate your claim. 


\title{
Concatenated Coding System with Iterated Sequential Inner Decoding
}

\author{
Ole Riis Jensen (riis@it.dtu.dk) \& Erik Paaske (ep@it.dtu.dk) \\ Institute of Telecommunication, Technical University of Denmark, DK-2800 Lyngby, Denmark
}

Abstract - We describe a concatenated coding system with iterated sequential inner decoding. The system uses convolutional codes of very long constraint length and operates on iterations between an inner Fano decoder and an outer Reed-Solomon decoder.

\section{INTRODUCTION}

We consider a concatenated system with a convolutional inner code, a block interleaver of degree I, and I outer RS codes of the same length, but with different redundancies/error correcting capabilities. After encoding by the outer codes and interleaving, the frame is split into a number of subframes. These are encoded by a memory $M$ convolutional code, which is terminated by $M$ input zeroes. The decoding for the inner code is performed by a number of sequential Fano decoders, which perform forward and backward (i. e. starting from the end of the subframe) decoding simultaneously, and on all the subframes in parallel. The process is monitored such that decoded symbols from the inner code in each of the RS words are counted. The non-decoded symbols are treated as erasures.

In a chosen implementation we use $\mathrm{I}=8$, the error correctional profile for the outer code [16 5016661666 ], and $M=23$. The three first decoders, and the 6th RS decoders are errors-and-erasure decoders which can correct e erasures as long as $e+2 t \leq 100$ and $e \leq 68$, respectively $\mathrm{e}+2 \mathrm{t} \leq 32$ and $\mathrm{e} \leq 16$. The other RS words are errorsonly decoders.

The first RS decoding attempt is then performed on the second RS word when 187 decoded symbols in this word are available from the inner decoders, and in case of a decoding failure (more than 50 errors detected) a new attempt is performed each time a new decoded symbol is available from the inner decoders. When the word is decoded the result is fed back and used to guide the sequential decoders in the continued decoding, i.e. the sequential decoders are forced to follow paths in the tree which agree with the RS decoded data. Decoding more and more RS words and feeding the results back to the inner decoders will in this way iterate the process towards a succesful decoding of the full frame. If 3 consequetive RS words ( 24 bits $>M$ ) are decoded, the forced inner decoding will effectively split the full frame into sub-sub-frames of length 48 bits, which can be decoded independently in both directions. If the decoding in one of these is stuck, a jump to the next sub-sub-frame can be made with only a small penalty.

\section{RESULTS FOR ITERATED SEQUENTIAL DECODING}

In a system where sequential decoding is used the code should have a good (or optimum) distance profile (DP) together, of course, with a large free distance. However, if decoding is performed forward and backward on a frame (or subframe) a suitable code must also have a good distance profile in its reversed form, since this is the code used in the backward decoding. From a code search we obtained the following ODP memory $M=23, d_{f}=54$ code written in hexadecimal form

$$
\mathrm{G}=[\text { 96A77B B7EA67 D0A25D E1C4D9 ] }
$$

which also has a very good DP in reversed form.

In the simulations we have used an AWGN channel quantized into 16 levels, and the quantizing thresholds are $\mathrm{E}_{\mathrm{s}} / \mathrm{N}_{\mathrm{o}}$-dependent as is common practice. The Fano decoders use an ordinary FANO metric, which in our case is unquantized ( 32 bit floating-point words). As a preliminary value of $\Delta$ we have used the ratio $\Delta / \mathrm{bm}_{\max }=6$, where $\mathrm{bm}_{\max }$ is the maximum branch metric. We have chosen to use interleaving degree $I=8$, but a further gain may be available by increasing the interleaving degree. A good choise for the number of subframes was determined through simulations to be 15 . The choice of profile (and rate) for the outer codes is by no means obvious and deserves further investigation. Our simulation approach includes a number of different profiles, and the one chosen here, has proven the best results. Very good profiles with only two different outer codes does also exist.

In Figure 1 we have shown the average number of computations $\mathrm{C}_{\mathrm{av}}$ found by simulation runs of 1000 frames (a total of $14,368,000$ information bits) with different signal to noise ratios. No errors appeared at all. The $\mathrm{E}_{\mathrm{b}} / \mathrm{N}_{0}$ values specified are the net values for the entire system. Since the overall rate of the system is $R_{\text {overall }}=0.216$ (including the small loss introduced by the termination of subframes) we notice that the inner sequential decoder operates at an $\mathrm{E}_{\mathrm{s}} / \mathrm{N}_{\mathrm{o}}$ which is $6.66 \mathrm{~dB}$ below $\mathrm{E}_{\mathrm{b}} / \mathrm{N}_{\mathrm{o}}$. With a computational cut-off rate $\mathrm{R}_{\text {comp }}$ that falls below the convolutional code rate of $1 / 4$ for $E_{b} / N_{0}<2.55 \mathrm{~dB}$ these results support our claim that a sequential decoder can operate well above $R_{\text {comp }}$ if some kind of side information is available. In this case the side information is achieved by using an outer code.

We notice that for $\mathrm{E}_{\mathrm{b}} / \mathrm{N}_{\mathrm{o}}=1.0 \mathrm{~dB}$ we can build a decoder with a $\mathrm{C}_{\mathrm{av}}$ that is at least 100 times smaller than the 16,384 decoding operations used by the Viterbi decoder for the $n=4, M=14$ code used in the Galileo mission. For $\mathrm{E}_{\mathrm{b}} / \mathrm{N}_{\mathrm{o}}=1.0 \mathrm{~dB}$ no frame required more than 2000 computations per bit and very few frames required more than 500 computations per bit. When we consider the $\mathrm{E}_{\mathrm{b}} / \mathrm{N}_{\mathrm{o}}=0.6 \mathrm{~dB}$ case $3 \%$ of the frames requires more than 10,000 computations, and $2 \%$ of the frames requires more than the Galileo code Viterbi decoder.

\section{CONCLUSION}

We have described a very efficient scheme utilizing iterated sequential decoding. However, the number of computations depends on the profile chosen and on the strategy used for the inner decoding, but as demonstated, very good results can be obtained.

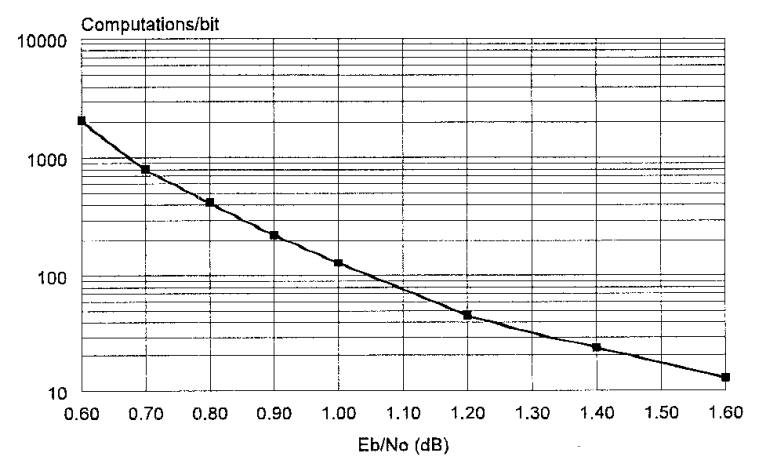

Figure 1: Average number of computations per bit as function of $\mathrm{E}_{\mathrm{b}} / \mathrm{N}_{0}$. 